\title{
Effectiveness of insecticidal nets on uncomplicated clinical malaria: a case-control study for operational evaluation
}

\author{
Georgia Barikissou Damien², Armel Djènontin²,3 Evelyne Chaffa ${ }^{4}$, Sandra Yamadjako ${ }^{2}$, Papa Makhtar Drame ${ }^{1,2,5}$,
} Emmanuel Elanga Ndille ${ }^{2}$, Marie-Claire Henry ${ }^{2}$, Vincent Corbel ${ }^{1,2}$, Franck Remoué ${ }^{1,2}$ and Christophe Rogier ${ }^{6,7,8}$

\begin{abstract}
Background: In a context of large-scale implementation of malaria vector control tools, such as the distribution of long-lasting insecticide nets (LLIN), it is necessary to regularly assess whether strategies are progressing as expected and then evaluate their effectiveness. The present study used the case-control approach to evaluate the effectiveness of LLIN 42 months after national wide distribution. This study design offers an alternative to cohort study and randomized control trial as it permits to avoid many ethical issues inherent to them.

Methods: From April to August 2011, a case-control study was conducted in two health districts in Benin; OuidahKpomasse-Tori (OKT) in the south and Djougou-Copargo-Ouake (DCO) in the north. Children aged 0-60 months randomly selected from community were included. Cases were children with a high axillary temperature $\left(\geq 37.5^{\circ} \mathrm{C}\right)$ or a reported history of fever during the last $48 \mathrm{~h}$ with a positive rapid diagnostic test (RDT). Controls were children with neither fever nor signs suggesting malaria with a negative RDT. The necessary sample size was at least 396 cases and 1188 controls from each site. The main exposure variable was "sleeping every night under an LLIN for the 2 weeks before the survey" (SL). The protective effectiveness (PE) of LLIN was calculated as PE $=1$ - odds ratio.

Results: The declared SL range was low, with 17.0 and $27.5 \%$ in cases and controls in the OKT area, and 44.9 and $56.5 \%$ in cases and controls, in the DCO area, respectively. The declared SL conferred $40.5 \%$ (95\% Cl 22.2-54.5\%) and $55.5 \%$ (95 \% Cl 28.2-72.4\%) protection against uncomplicated malaria in the OKT and the DCO areas, respectively. Significant differences in PE were observed according to the mother's education level.

Conclusion: In the context of a mass distribution of LLIN, their use still conferred protection in up to $55 \%$ against the occurrence of clinical malaria cases in children. Social factors, the poor use and the poor condition of an LLIN can be in disfavour with its effectiveness. In areas, where LLIN coverage is assumed to be universal or targeted at high-risk populations, case-control studies should be regularly conducted to monitor the effectiveness of LLIN. The findings will help National Malaria Control Programme and their partners to improve the quality of malaria control according to the particularity of each area or region as far as possible.
\end{abstract}

Keywords: Effectiveness, Case-control study, Evaluation, Malaria, LLIN

\section{Background}

Malaria remains a public health problem in sub-Saharan Africa despite massive control measures deployed over

\footnotetext{
*Correspondence: barikiss2000@yahoo.fr

${ }^{2}$ IRD-UMR MIVEGEC (IRD224-CNRS5290-Universités Montpellier 1 et 2),

Centre de Recherche Entomologique de Cotonou (CREC), Cotonou, Benin

Full list of author information is available at the end of the article
}

the last several years. The most vulnerable populations are children under 5 years of age [1]. Many countries have focused their efforts on the Roll Back Malaria strategies, such as coverage and use of long-lasting insecticide-treated nets (LLIN), indoor residual spraying (IRS) if appropriate, early case detection with the rapid diagnostic test (RDT), and prompt appropriate treatment by artemisinin-based combination therapy (ACT) [2]. 
Between 2000 and 2010, the World Health Organization (WHO) recorded reductions of more than $50 \%$ in the health centre-reported cases in 43 of the 99 countries endemic for malaria, showing major progress [3].

LLIN remains the most common and effective tool used to prevent malaria [4]. Between 2004 and 2013, the ownership rate of LLIN strongly increased from 5 to $67 \%$ [4]. Some randomized control studies have already shown that LLIN implementation leads to significant protection against malaria infection, morbidity, and mortality $[5,6]$. For the purpose of reducing malaria case incidence rates by $75 \%$ by 2015 , operational research is needed to understand effectiveness of vector control interventions [4].

In Benin, large-scale coverage of LLIN using free distribution has been implemented since 2007. Nevertheless the number of reported cases of malaria in 2012 remained high with confirmed 1,365,389 cases in the general population of which 601,347 were children under 5 years of age [7]. The proof of malaria morbidity reduction has become the major objective of the National Malaria Control Programme (NMCP) in Benin, and few studies at the community level have been conducted on this subject [7]. Therefore, some questions still need answers in Benin. For example are malaria control strategies effective? What about LLIN effectiveness in the context of vectors resistance to pyrethroid insecticide? Do the investigators currently have adequate and operational indicators to evaluate malaria control tools such as LLIN in the context of universal coverage?

To evaluate the effectiveness of LLIN, case-control studies offers an alternative to cohort study and randomized control trial as it permits to avoid many ethical issues inherent to them. This type of epidemiological study has already been used for the evaluation of interventions (vaccines, vector control tools) against other infectious diseases, because it avoids many of the ethical issues inherent in longitudinal and experimental studies [8-13]. In the current context of wide distribution of LLIN, it is not possible to compare a group without LLIN coverage and a group with LLIN using a phase III trial. A case-control study can also be less expensive and more operationally feasible than longitudinal studies. In the present study, the main objective was to use a case-control design to evaluate the effectiveness of LLIN on uncomplicated malaria in children, using the main exposure variable defined as "sleeping every night under an LLIN for the 2 weeks before the survey" (SL) and, taking into account epidemiological and social information related to malaria control.

\section{Methods}

\section{Study design}

A matched case-control study was carried out in the Ouidah-Kpomasse-Tori Bossito (OKT), and
Djougou-Copargo-Ouake (DCO) rural health districts located in Benin, West Africa (Fig. 1). In both districts, LLINs were freely distributed to pregnant women and children aged from 0 to 5 years by the NMCP in October 2007 by national campaign. Permanent free distribution has been provided by maternity during pre natal consultations and during vaccination for children aged 9 months. The study area was free of indoor residual spraying campaign. Pyrethroid resistance was widely reported in African malaria vectors especially in Benin $[14,15]$. In the study areas (Figs. 2,3) vector resistance to pyrethroid insecticides was detected simultaneously; the mortality rate of vectors after exposure to permethrin and deltamethrin were 20 and $60 \%$ respectively in OKT, 10 and $36 \%$ in DCO (unpublished data) [16]. The characteristics of these areas have been described previously [16-18]. The inclusion criteria for villages were population size 1000-1800 inhabitants, with the objective of observing at least 15 malaria cases per village. The target population was children aged 0-60 months, living in the selected villages, whose parents gave their informed consent. At least 30 villages were randomly selected in each health district. At first all the children aged 0-60 months were included in the study. After determining the clinical status, "neither case nor control" children were excluded (Fig. 4). Cases and controls were matched by village of residence (1:3). A case was defined as a child with a (i) high axillary temperature $\left(\geq 37.5^{\circ} \mathrm{C}\right)$ or (ii) a history of fever as reported by the mother or guardian during the $48 \mathrm{~h}$ preceding the day of blood sampling, whatever the association with other signs suggesting malaria (sweats, shivers, headaches, nausea, or vomiting) but associated with a positive RDT. A control was defined as a child who had no fever or signs suggesting malaria with a negative RDT. The CareStart ${ }^{\text {TM }}$ RDT used detected histidinerich protein-2 (HPR2) specific to Plasmodium falciparum.

\section{Ethical clearance}

Ethics clearance for the study was obtained from the National Ethical Committee for Medical Research in Benin (CNERS, Number 003, 24 March 2011, Institutional Review Board No. 00006860) and Institut de Recherche pour le Développement (IRD)'s consultative committee for deontology and ethics (CCDE). All sick children, whether or not they were participating in the study, were treated by medical staff based on an integrated management of childhood illness strategy during the investigation. Specifically, the malaria cases were treated with artemether-lumefantrine, according to NMCP recommendations in Benin.

\section{Sample size}

The sample size was calculated under the assumption of $30 \%$ protection conferred by using $\mathrm{LN}$, as previously 


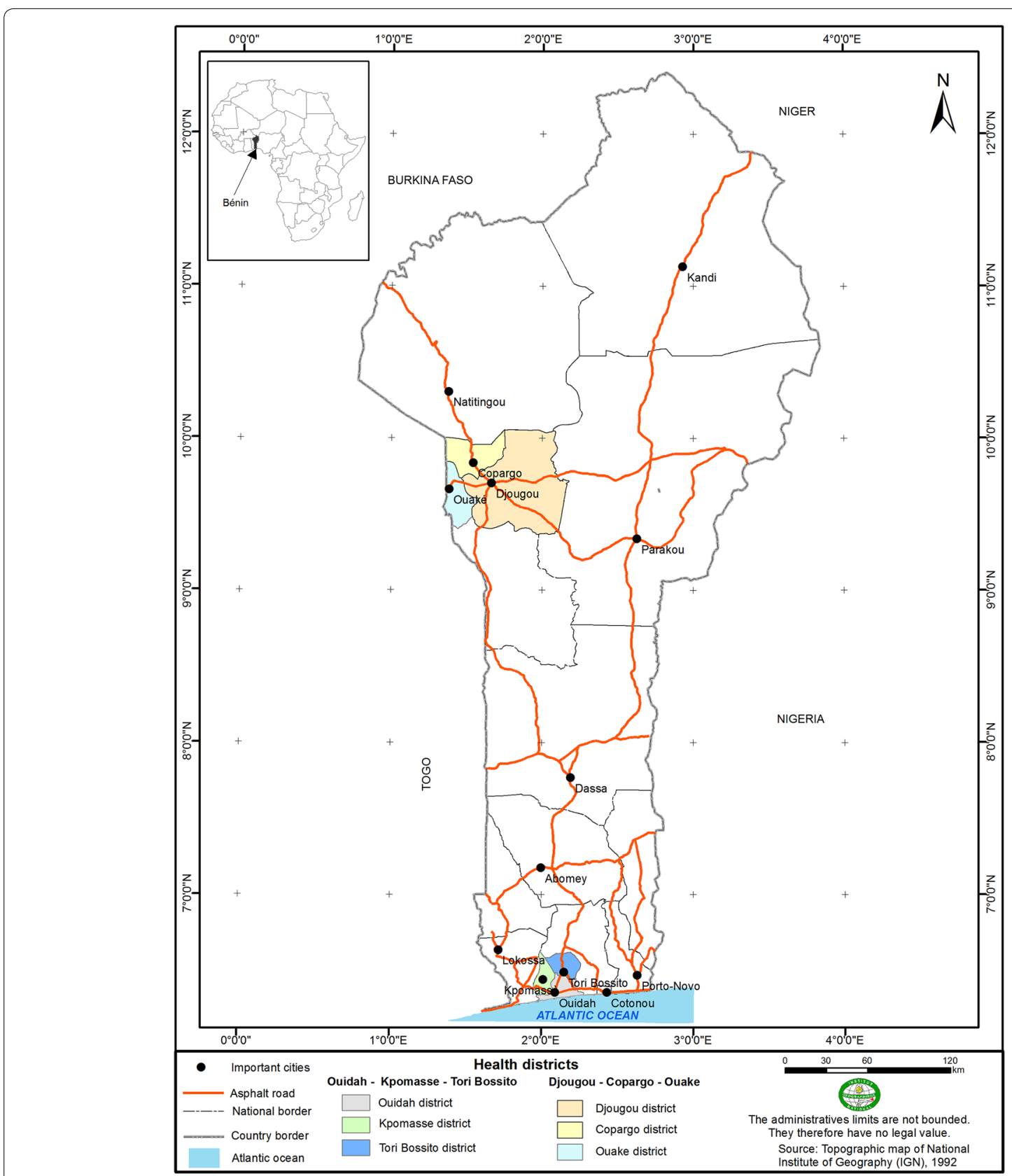

Fig. 1 Map of Republic of Benin (RB) located in West Africa. Ouidah-Kpomasse-Tori Bossito and Djougou-Copargo-Ouake health districts were the study area

observed in southern Benin in prevalence of infection [18]. The sample size formula used was: $N=[p \times q$ $\left.(1+1 / c)\left(Z_{\alpha}+Z_{2 \beta}\right)^{2}\right] \times$ cluster effect $/\left(p_{0}-p_{1}\right)^{2}[12]$, where $\mathrm{N}$ is the number of cases, $\mathrm{p}_{0}=50 \%$ is the proportion of persons using LLIN among the controls, $\mathrm{p}_{1}=33 \%$ is the proportion of persons using LLIN among the cases, $\mathrm{p}=45.75 \%$ is the proportion of persons using LLIN in the whole sample, $q=1-p, c=3$ is the number of controls per case, $\mathrm{Z}_{\alpha}=1.645$ for a unilateral alpha risk of $5 \%$ and $Z_{2 \beta}=0.84$ for a power at $80 \%$, and with a cluster effect of 1.5. Under these assumptions, the required sample size was 396 cases and 1188 controls per site.

\section{Procedure}

Data were collected over 6 weeks in the rainy season (peak of malaria transmission), from April to May 2011 


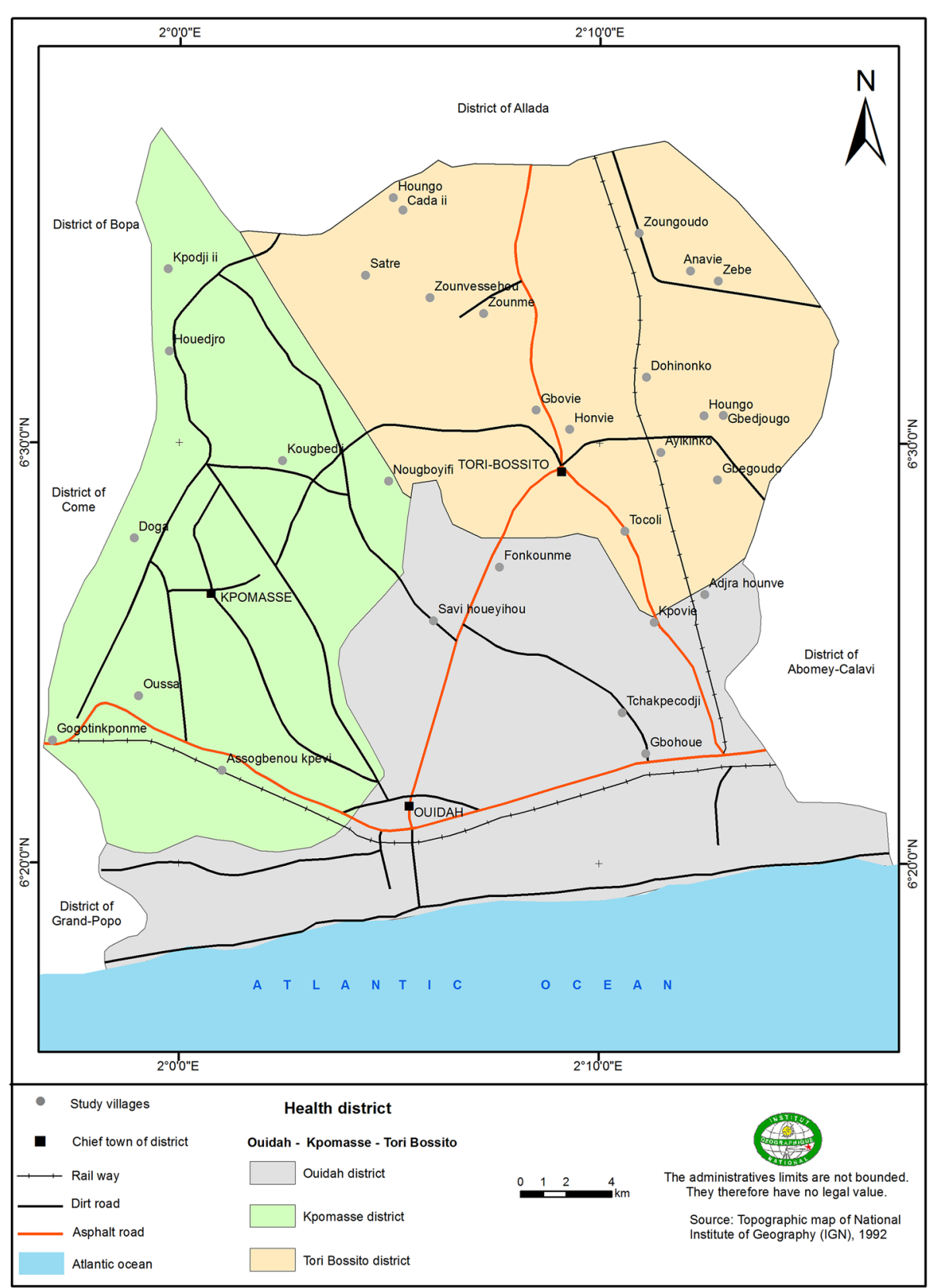

Fig. 2 Study villages located in Ouidah-Kpomasse-Tori Bossito health district in southern Benin. A total of 31 villages were included

in the OKT district and from July to August 2011 in the DCO district using a cross-sectional survey. Cases and controls were identified by medical staff and by active detection in community in order to identify all children in each village (Figs. 2, 3). CareStart ${ }^{\mathrm{TM}}$ RDTs belonged to those certified and recommended by WHO and were assumed to have a $100 \%$ sensitivity and a $100 \%$ specificity for a parasite density at 2000 parasites/ $\mu \mathrm{L}$ [19]. All children were tested by RDT. When the result of the RDT was invalid, it was cancelled and re-tested. Cases and controls were randomly selected with equal probability of inclusion per village. The following day after clinical status determination and RDT testing, the mothers or the guardians of the children were questioned face-to-face by trained interviewers to obtain demographic information and determine the use of bed nets and other prevention tools. A standardized questionnaire was administrated under the same conditions for cases and controls by the 


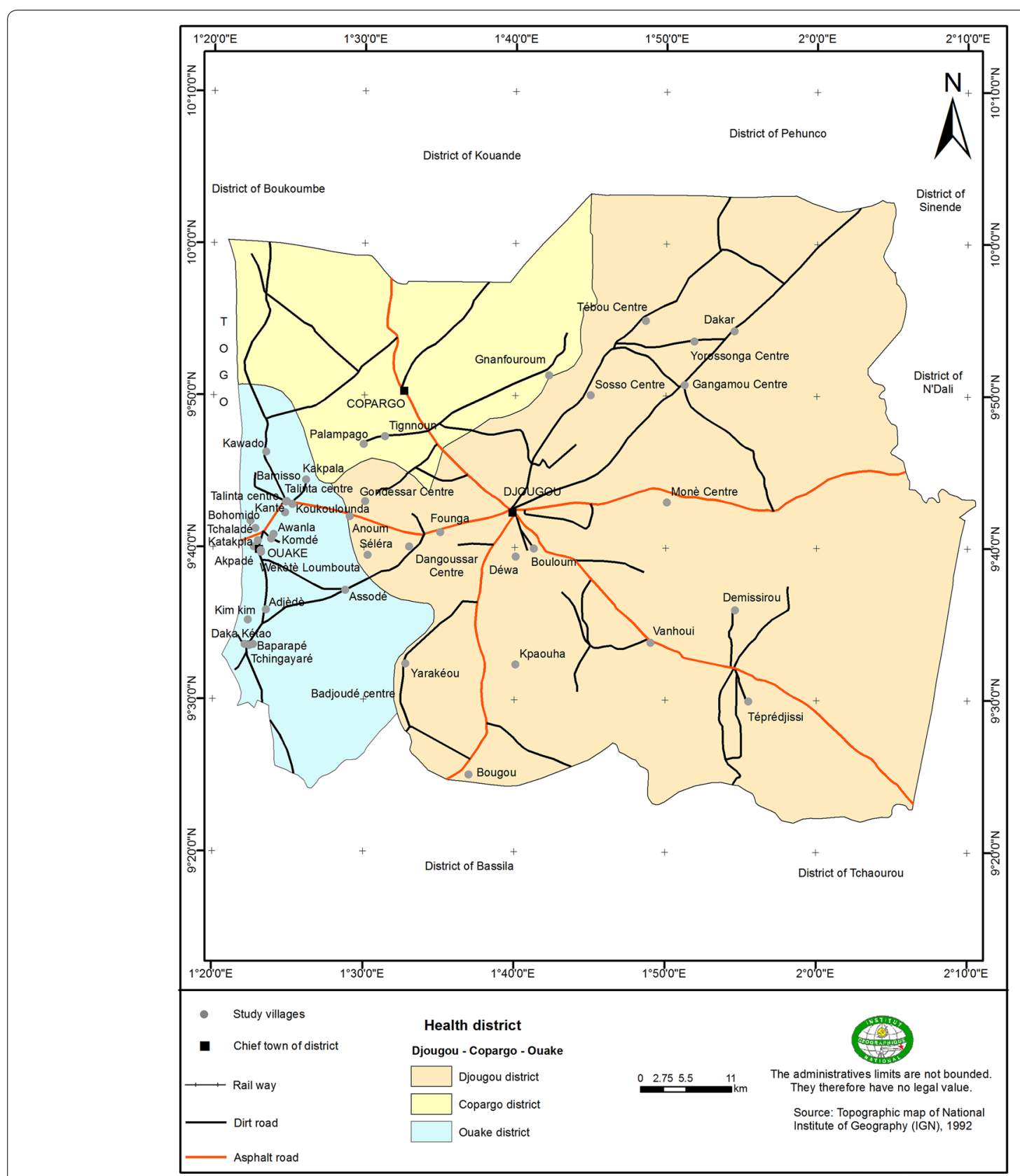

Fig. 3 Study villages located in Djougou-Copargo-Ouake health district in northern Benin. A total of 42 villages were included

same investigators to collect information concerning the use of LLIN. Interviewers were not informed of the clinical status of the children. Questionnaires were verbally administrated in French or in the local language. When the mother or a guardian was absent, the team came back the next day to administer the questionnaire. When the mother or guardian was not found the second day, the child was considered as lost to follow-up. Data collection was supervised daily and systematically. An unexpected quality control was periodically undertaken on the data collection in the field and on $10 \%$ of the RDTs.

\section{Measurement of exposure variables}

The principal exposure variable was SL considering the 14-day incubation period of $P$. falciparum. A LLIN was sought and verified de visu (in the hung position) by the interviewers during the survey. Two categories of responses were defined, i.e. "always" and "not always" 


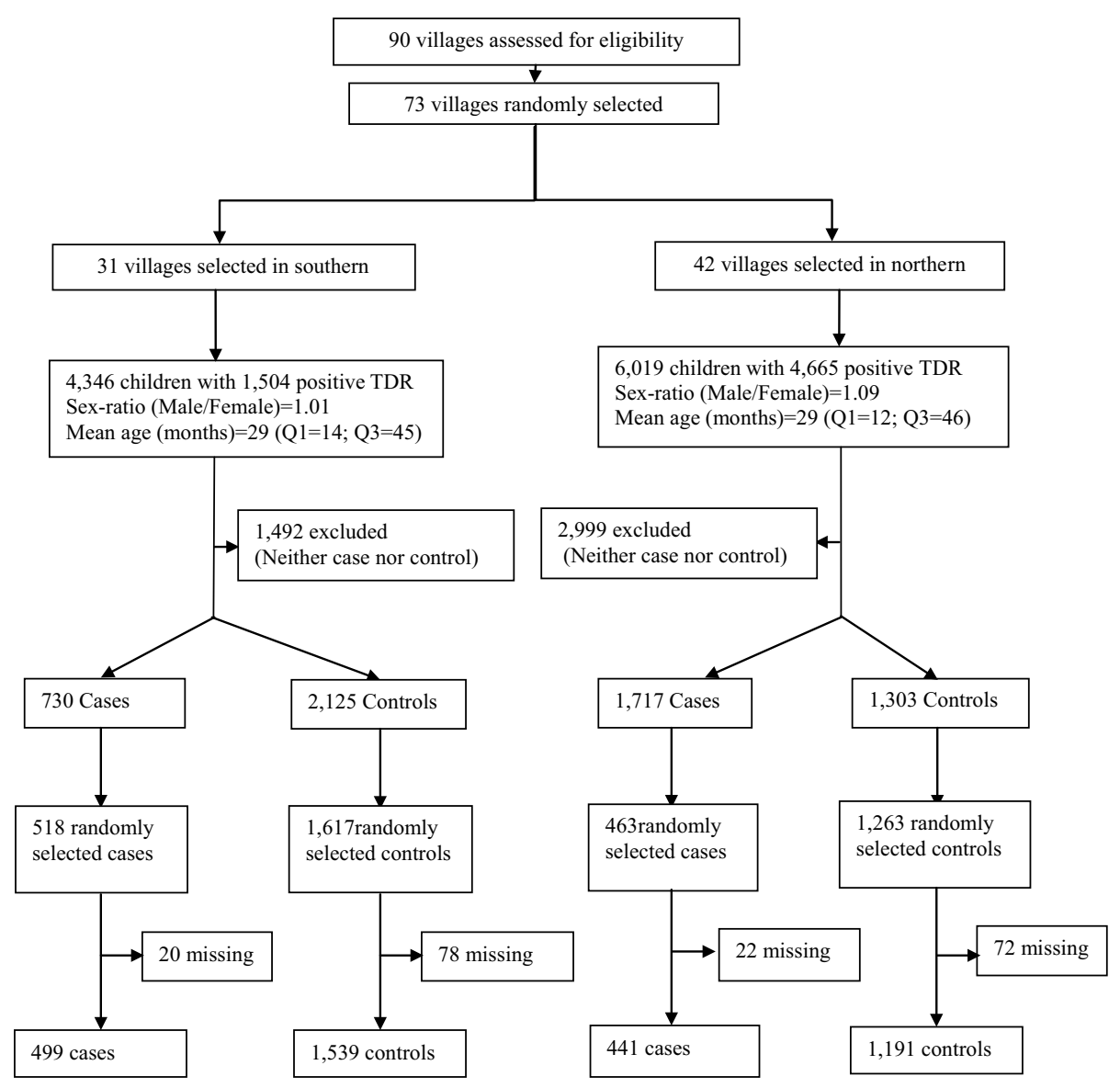

Fig. 4 Study profile. Cases were children with a high axillary temperature $\left(\geq 37.5^{\circ} \mathrm{C}\right)$ or a reported history of fever during the last $48 \mathrm{~h}$ with a positive rapid diagnostic test (RDT). Controls were children with neither fever nor signs suggesting malaria with a negative RDT. Neither case nor control were (i) children with a high axillary temperature $\left(\geq 37.5^{\circ} \mathrm{C}\right)$ or a reported history of fever during the last $48 \mathrm{~h}$ with a negative RDT and (ii) children with no signs suggesting malaria associate to positive RDT

used. The modality "not always" included the answers "sometimes" and "never" used. The analysis also included variables for controlling the following potential confounding factors: use of other tools than LLIN for protection against mosquito bites (coil, domestic insecticide spray, smoking with traditional grass), use of drugs perceived to be malaria chemoprophylaxis during the previous 2 weeks (herbal tea, paracetamol, and chloroquine), child's age in two groups (0-23 or 24-60 months), sex, the time the child went to bed the previous night (at night fall, before 10 p.m. and after 10 p.m.), mother's or guardian's age grouped as $<25,25-35$, and $>35$ years old, mother's education level (at least primary school or no education), mother's economic activity, number of dependent children $(1,2,3$, or $\geq 4)$, mother's or guardian's knowledge of malaria transmission (i.e. malaria is transmitted by mosquito bites) and prevention (i.e. mosquito nets can prevent malaria). The LN condition was assessed by the age of the net from the beginning of its use ( $\leq 3$ or $>3$ years), physical condition (with or without holes), and cumulative number of washings ( $\leq 20$ or $>20$ washes). The age of the $\mathrm{LN}$ was estimated using time references (periods of mass distribution and age of children for whom the $\mathrm{LN}$ was given).

\section{Statistical analysis}

All data were entered in the field and checked for errors using the Access 2003 program. The data were analysed using SAS version 9.3 (SAS Institute Inc., Cary, NC, USA). The proportions of cases and controls according to exposure variables were calculated. A sample of 1:3 case-controls matched in the village of residence in the OKT and DCO health districts was considered. The adjusted matched odds ratio (OR) and $95 \%$ confidence interval (CI) for calculation of $\mathrm{LN}$ effectiveness were estimated using conditional logistic regression and taking into account the cluster random effect [20,21]. Variables associated with clinical uncomplicated malaria with a $p$ 
value $<0.25$ in univariate analysis were included in multivariate analysis. For all of the analysis, the level of significance was set at 0.05 . LN effectiveness as protective efficacy (PE) was calculated as: $(1-$ matched OR $) \times 100$.

\section{Results}

A total of 90 villages were eligible for the study. Seventythree of them were randomly selected, 31 in the OKT health district and 42 in the DCO district. Details of the study profile are described in Fig. 4. Finally, 499 cases and 1539 controls in the OKT district and 441 cases and 1191 controls in the DCO district were enrolled in the investigation.

The characteristics of the cases and controls included are shown in Tables 1 and 2, according to child, LLIN, and mother or guardian characteristics. Overall, the controls were younger in age than the cases in both health districts $(\mathrm{p}<0.0001)$ with no significant difference according to sex. In the OKT district, $17.0 \%$ of the cases and $27.5 \%$ of the controls had slept under a LLIN every night during the previous 2 weeks compared to $44.9 \%$ of cases and $56.5 \%$ of the controls in the DCO district.

In the OKT health district (southern Benin), in univariate analysis, the variable SL was significantly associated with uncomplicated malaria episodes [OR 0.54 (95\% CI $0.42-0.70) ; \mathrm{p}<0.0001]$. The use of an LLIN is lower in the case group than in the control. Other factors were significantly associated with uncomplicated malaria in the two districts. Age group (0-23 months) of children ( $p<0.0001)$, mother's or guardian's level of education at least primary school $(\mathrm{p}=0.0337)$, time the children went to bed the previous night (between nightfall and 10 p.m. and after 10 p.m.) versus at nightfall $(\mathrm{p}<0.0001)$, and age of LLIN under 3 years $(\mathrm{p}=0.0018)$ were uncomplicated malaria protection factors. Some factors were significantly associated with increasing of uncomplicated malaria episodes: (i) use of prophylactic treatments against malaria ( $\mathrm{p}=0.0063)$, (ii) use other anti-mosquito measures ( $\mathrm{p}=0.0003$ ), (iii) age of mother, or guardian ( $\geq 35$ versus $25-35, p=0.0001$ ), and (iv) number of children in the household ( $\mathrm{p}=0.0054$; Table 1). In the DCO health district (northern Benin), in univariate analysis, the variable SL was significantly associated with decrease of uncomplicated malaria episodes [OR 0.63 (95 \% CI $0.50-0.78) ; \mathrm{p}<0.0001$ ] as it was for OKT district. Other factors significantly associated with decreasing of uncomplicated malaria were: (i) age group (0-23 months) of children ( $\mathrm{p}<0.0001$ ), (ii) age of LLIN (under 3 years) ( $p=0.0269$ ), (iii) mother's or guardian's level of education at least primary school ( $\mathrm{p}=0.0059)$, and (iv) mother's knowledge that "mosquitoes transmit malaria" $(\mathrm{p}=0.0246)$. The use of prophylactic treatments against malaria $(\mathrm{p}=0.0418)$ and mother's or guardian's age of mother, or guardian ( $\geq 35$ vs $25-35, \mathrm{p}<0.0001)$ were associated with increasing of uncomplicated malaria episodes in DCO district (Table 2). In multivariate analysis, the variable SL was a significant protective factor against uncomplicated clinical malaria episodes, with $\mathrm{PE}=0.41$ (95 \% CI 0.22-0.55) in the OKT district (Table 3). There was a significant interaction $(\mathrm{p}=0.0233)$ between the effects of the mother's or guardian's level of education and the use of an LLIN in the DCO district. Among children whose mother had at least a primary school level of education, PE was 0.55 (95\% CI 0.28-0.72), and it was 0.16 (95\% CI -0.10 to 0.36 ) in the other children, suggesting a loss of effectiveness of LLIN when mothers or guardians of children had never attended school in the DCO (Table 4).

\section{Discussion}

This community-based case-control study was used to evaluate the effectiveness of LLIN in a context of wide distribution. When considering 1:3 case-controls, matching on the village of residence, "sleeping every night under an LLIN" conferred $40 \%$ protection from malaria episodes in southern Benin (OKT) and up to $55 \%$ in northern Benin (DCO) among children. The mother's or guardian's level of education was a significant modifier of the effectiveness of LLIN. This modification effect was only observed in the DCO district despite the similar frequency of mothers' and guardians' primary school level of education among controls in both districts: $32 \%$ in the OKT and $29 \%$ in the DCO. In the present study, use of an LLIN was low (17-57 \%) and far from the $80 \%$ expected and which can give mass effect $[4,22]$. In addition, malaria vectors were resistant to pyrethroïd insecticides. The mortality rate of vectors after exposure to these insecticides was very low, under $90 \%$ required [23]. Nevertheless, the PE of an LLIN was between 40 and $50 \%$. An LLIN can then contribute to reduce malaria case incidence as expected [4].

This study identified other factors significantly associated with uncomplicated clinical malaria. The main risk factors of malaria episodes were the use of drugs perceived to be malaria prophylactic treatments (herbal tea, paracetamol, and chloroquine) and the use of antimosquito measures other than bed nets (coil, domestic insecticide spray, and smoking with traditional grass). The use of herbal tea, paracetamol, and chloroquine for children as prophylactic drugs was very high. This habit can lead to temporarily diminution or disappearance of fever but not clean the parasites. In the same way, the use of anti-mosquito measures other than bed nets may reduce malaria vector biting but the risk of malaria infection and morbidity remains. However, the use of these non-recommended malaria prevention measures could 
Table 1 Factors associated with uncomplicated clinical malaria, Ouidah-Kpomasse-Tori Bossito health district

\begin{tabular}{|c|c|c|c|c|}
\hline Variables & $\mathrm{N}$ case & $\mathrm{N}$ control & Crude OR (95 \% Cl) & p value \\
\hline \multicolumn{5}{|l|}{ Information about children } \\
\hline \multicolumn{5}{|c|}{ Sleeping every night under an LLIN for the 2 weeks before the survey } \\
\hline Sometimes or never & 414 & 1116 & 1 & \\
\hline Every night & 85 & 423 & $0.54(0.42-0.70)$ & $<0.0001$ \\
\hline \multicolumn{5}{|l|}{ Age group (month) } \\
\hline $24-60$ & 394 & 809 & 1 & \\
\hline $0-23$ & 105 & 730 & $0.29(0.23-0.37)$ & $<0.0001$ \\
\hline \multicolumn{5}{|l|}{ Sex } \\
\hline Male & 252 & 776 & 1 & \\
\hline Female & 247 & 763 & $0.99(0.82-1.23)$ & 0.9756 \\
\hline \multicolumn{5}{|c|}{ Use of drugs perceive to be prophylactic treatment against malaria } \\
\hline No & 126 & 488 & 1 & \\
\hline Yes & 373 & 1051 & $1.37(1.09-1.73)$ & 0.0063 \\
\hline Hour of sleeping last night & & & & $<0.0001$ \\
\hline At night fall & 354 & 933 & 1 & \\
\hline Between night fall and 10 p.m. & 123 & 478 & $0.68(0.54-0.86)$ & 0.0011 \\
\hline After 10 p.m. & 22 & 128 & $0.45(0.28-0.72)$ & 0.0009 \\
\hline \multicolumn{5}{|l|}{ Use other anti-mosquito measures } \\
\hline Never & 317 & 1109 & 1 & \\
\hline Every day or sometimes & 182 & 430 & $1.48(1.20-1.83)$ & 0.0003 \\
\hline \multicolumn{5}{|l|}{ Information about mosquito net } \\
\hline \multicolumn{5}{|l|}{ Age (year) } \\
\hline$>3$ & 156 & 373 & 1 & \\
\hline$\leq 3$ & 343 & 1166 & $0.70(0.56-0.88)$ & 0.0018 \\
\hline \multicolumn{5}{|l|}{ Number of times washed since obtained } \\
\hline$>20$ & 111 & 333 & 1 & \\
\hline$\leq 20$ & 388 & 1206 & $0.96(0.76-1.23)$ & 0.7742 \\
\hline \multicolumn{5}{|l|}{ Holes } \\
\hline No & 251 & 744 & 1 & \\
\hline Yes & 248 & 795 & $0.92(0.76-1.13)$ & 0.4444 \\
\hline \multicolumn{5}{|l|}{ Information about mother or guardian } \\
\hline Age (year) & & & & 0.0004 \\
\hline$<25$ & 110 & 365 & $1.07(0.83-1.38)$ & 0.6121 \\
\hline $25-35$ & 234 & 830 & 1 & \\
\hline$>35$ & 155 & 344 & $1.60(1.26-2.03)$ & 0.0001 \\
\hline \multicolumn{5}{|l|}{ Primary school at least } \\
\hline No & 365 & 1048 & 1 & \\
\hline Yes & 134 & 491 & $0.78(0.63-0.98)$ & 0.0337 \\
\hline Profit-making activity & & & & 0.4144 \\
\hline Farming/breeding/fishing/handicraft & 219 & 645 & 1 & \\
\hline Employee status/shopkeeper & 269 & 844 & $1.54(0.79-3.01)$ & 0.2046 \\
\hline No activity & 11 & 50 & $1.45(0.74-2.82)$ & 0.2761 \\
\hline Number of children in the household & & & & 0.0054 \\
\hline 1 & 57 & 252 & 1 & \\
\hline 2 & 107 & 362 & $1.31(0.91-1.87)$ & 0.1456 \\
\hline 3 & 105 & 337 & $1.38(0.96-1.98)$ & 0.0834 \\
\hline$\geq 4$ & 230 & 588 & $1.73(1.25-2.40)$ & 0.0010 \\
\hline \multicolumn{5}{|l|}{ Knows mosquitoes transmit malaria } \\
\hline No & 10 & 25 & 1 & \\
\hline
\end{tabular}


Table 1 continued

\begin{tabular}{lcccc}
\hline Variables & N case & N control & Crude OR (95 \% Cl) & p value \\
\hline Yes & 489 & 1514 & $0.81(0.38-1.70)$ & \\
Knows mosquito net protection against malaria & & 105 & 1 & 0.5727 \\
No & 37 & 1434 & $0.91(0.62-1.35)$ & 0.6523 \\
Yes & 462 & & \\
\hline
\end{tabular}

Factors associated with uncomplicated clinical malaria were grouped into information (in italic text) about (i) children aged 0-60 months, (ii) mosquito net, and (iii) mother or guardian of children characteristics. Univariate conditional logistic regression taking into account cluster effect was used

be related to a perception of higher risk of malaria and therefore could be associated with the risk of clinical malaria.

Protective factors of uncomplicated malaria episodes were the child's age $>24$ months, mother's education level (at least primary school), and knowing of the role mosquitoes play in the transmission of malaria and of the protection of LLIN against malaria.

Social factor as school level has already known to be a determinant of malaria case occurrence $[24,25]$. Other determinants that were not taken into account in this study, and related to the social and economic environment, probably interfered in the both districts. To better understand the determinants of the effectiveness of LLIN in an area, additional and specific anthropological studies are therefore needed.

Some limitations to this study stem from the use of the case-control study design. The efficacy of interventions deployed can be defective. For example, if malaria vectors are locally resistant to insecticides used for treated nets or for wall spraying, or Plasmodium are locally resistant to anti-malarial drugs used for preventive measures or chemoprophylaxis, the effectiveness of interventions may be affected in some areas more than others. The estimation of an average effectiveness, without taking into account several heterogeneous areas in terms of vector and parasite sensitivity, would not be relevant or would not detect localized deficiencies in effectiveness.

The different case definitions for uncomplicated malaria have advantages and disadvantages. The best definition of uncomplicated clinical malaria is based on a parasite density threshold associated with signs or symptoms suggesting malaria $[18,26]$. The definition of clinical malaria cases in the present study was only based on the detection of Plasmodium infection by RDT, i.e. regardless of the parasite density. This definition could lack specificity [27]. Defining malaria attacks by the association of fever with a patent parasite infection (e.g., detected by a RDT) can overestimate the number of fevers caused by malaria (excess of false-positive results), even in areas of low endemicity. It may underestimate the PE of malaria interventions, especially as asymptomatic Plasmodium infections are more common in highly endemic areas than in areas of low endemicity and in the most immune compared to those who have not had exposure long enough to develop immunity. The use of parasite density to define malaria cases suitably at every level of endemicity and at all ages most often requires microscopy studies and therefore carries a higher cost and is a source of inter-study variability due to potential differences in reading blood smears. Although this definition avoids underestimating the effectiveness of LLIN and distortions in this estimation depending on malaria endemicity and the age of individuals, it does not reflect the burden of malaria as usually estimated by health systems.

This study has many valuable aspects nevertheless. The cases and controls were identified only in community concurrently from the same at-risk population. The health center recruitment may be a source of information bias, especially in terms of exposure variables. Since the LLIN were given at no cost, mothers may lie (prevarication bias) about their child's exposure, especially in the health center area when the children were sick. In this study, direct observation of the net in the hung position in the house was used, knowing that questions about the use of mosquito bed nets could lead to a deliberate false response.

The study had a very short duration. The positive effect of short-term use cannot be found in longitudinal or cohort studies, which may change the community's behavior. Given that individuals were asked the same questions several times, the quality of responses may be compromised, resulting in a high rate of LLIN use regardless of the child's clinical status [18]. The consequence is no difference in exposure between individuals and therefore problems demonstrating the effectiveness of LLIN due to the loss of statistical power. Concerning the case-control study, the difficulties finding cases and controls according to epidemiological malaria endemicity and season may extend the duration of the study.

The risk of malaria attacks is assumed to be higher about 2 weeks after inoculation of sporozoites by anopheline vectors. The cover of LLIN can mean the possession and/or the hung of LLIN in the household $[4,18,28]$. The 
Table 2 Factors associated with uncomplicated clinical malaria, Djougou-Copargo-Ouake health district

\begin{tabular}{|c|c|c|c|c|}
\hline Variables & $\mathrm{N}$ case & $\mathrm{N}$ control & Crude OR (95 \% Cl) & $\mathrm{p}$ value \\
\hline \multicolumn{5}{|l|}{ Information about children } \\
\hline \multicolumn{5}{|c|}{ Sleeping every night under an LLIN for the 2 weeks before the survey } \\
\hline Sometimes or never & 243 & 518 & 1 & \\
\hline Every night & 198 & 673 & $0.63(0.50-0.78)$ & 0.0001 \\
\hline \multicolumn{5}{|l|}{ Age group (month) } \\
\hline $24-60$ & 293 & 366 & 1 & \\
\hline $0-23$ & 148 & 825 & $0.22(0.18-0.28)$ & $<0.0001$ \\
\hline \multicolumn{5}{|l|}{ Sex } \\
\hline Male & 226 & 637 & 1 & \\
\hline Female & 215 & 554 & $1.09(0.88-1.36)$ & 0.4227 \\
\hline \multicolumn{5}{|c|}{ Use of drugs perceive to be prophylactic treatment against malaria } \\
\hline No & 300 & 871 & 1 & \\
\hline Yes & 141 & 320 & $1.28(1.01-1.62)$ & 0.0418 \\
\hline Hour of sleeping last night & & & & 0.2891 \\
\hline At night fall & 135 & 393 & 1 & \\
\hline Between night fall and 10 p.m. & 243 & 660 & $1.07(0.84-1.37)$ & 0.1169 \\
\hline After 10 p.m. & 63 & 138 & $1.33(0.93-1.90)$ & 0.5796 \\
\hline \multicolumn{5}{|l|}{ Use other anti-mosquito measures } \\
\hline Never & 326 & 890 & 1 & \\
\hline Every day or sometimes & 115 & 301 & $1.04(0.81-1.34)$ & 0.7401 \\
\hline \multicolumn{5}{|l|}{ Information about mosquito net } \\
\hline \multicolumn{5}{|l|}{ Age (year) } \\
\hline$>3$ & 86 & 178 & 1 & \\
\hline$\leq 3$ & 355 & 1013 & $0.73(0.55-0.96)$ & 0.0269 \\
\hline \multicolumn{5}{|l|}{ Number of times washed since obtained } \\
\hline$>20$ & 69 & 200 & 1 & \\
\hline$\leq 20$ & 372 & 991 & $1.09(0.81-1.47)$ & 0.5804 \\
\hline \multicolumn{5}{|l|}{ Holes } \\
\hline No & 143 & 360 & 1 & \\
\hline Yes & 298 & 831 & $0.90(0.72-1.14)$ & 0.3893 \\
\hline \multicolumn{5}{|l|}{ Information about mother or guardian } \\
\hline Age (year) & & & & $<0.0001$ \\
\hline$<25$ & 98 & 367 & $0.83(0.63-1.10)$ & 0.1969 \\
\hline $25-35$ & 181 & 564 & 1 & \\
\hline$>35$ & 162 & 260 & $1.94(1.50-2.52)$ & $<0.0001$ \\
\hline \multicolumn{5}{|l|}{ Primary school at least } \\
\hline No & 342 & 842 & 1 & \\
\hline Yes & 99 & 349 & $0.70(0.54-0.90)$ & 0.0059 \\
\hline Profit-making activity & & & & 0.6851 \\
\hline Farming/breeding/fishing/handicraft & 187 & 489 & 1 & \\
\hline Employee status/shopkeeper & 156 & 413 & $1.13(0.85-1.50)$ & 0.4058 \\
\hline No activity & 98 & 289 & $1.11(0.83-1.49)$ & 0.4719 \\
\hline Number of children in the household & & & & 0.1303 \\
\hline 1 & 75 & 265 & 1 & \\
\hline 2 & 93 & 232 & $1.42(0.99-2.01)$ & 0.0527 \\
\hline 3 & 95 & 229 & $1.47(1.03-2.08)$ & 0.0328 \\
\hline$\geq 4$ & 178 & 465 & $1.35(0.99-1.84)$ & 0.0554 \\
\hline \multicolumn{5}{|l|}{ Knows mosquitoes transmit malaria } \\
\hline No & 131 & 304 & 1 & \\
\hline
\end{tabular}


Table 2 continued

\begin{tabular}{lcccc}
\hline Variables & N case & N control & Crude OR (95 \% Cl) & p value \\
\hline Yes & 310 & 887 & $0.75(0.58-0.96)$ & \\
Knows mosquito net protection against malaria & & & & \\
No & 121 & 263 & 1 & 0.0246 \\
Yes & 320 & 928 & $0.81(0.64-1.03)$ & 0.0910 \\
\hline
\end{tabular}

Factors associated with uncomplicated clinical malaria were grouped into information (in italic text) about (i) children aged 0-60 months, (ii) mosquito net, and (iii) mother or guardian of children characteristics. Univariate conditional logistic regression taking into account cluster effect was used

Table 3 Association between uncomplicated clinical malaria and sleeping under an LLIN adjusted for other variables, Ouidah-Kpomasse-Tori Bossito health district

\begin{tabular}{lc}
\hline Variables & Adjusted OR $\mathbf{9 5} \% \mathbf{C l})$ \\
\hline $\begin{array}{l}\text { Sleeping every night under an LLIN for the } 2 \text { weeks before the survey } \\
\text { Sometimes or never }\end{array}$ & 1 \\
Every night & $0.59(0.45-0.78)^{\mathrm{a}}$ \\
Children age group (month) & 1 \\
$24-60$ & $0.31(0.24-0.40)$ \\
$0-23$ & $1.35(1.03-1.78)$ \\
Mother or guardian age group (year) & 1 \\
$<25$ & $1.37(1.07-1.75)$ \\
$25-35$ & 1 \\
$\geq 35$ & $0.77(0.61-0.97)$ \\
Mosquito net age group (year) & 1 \\
$>3$ & $1.29(1.02-1.63)$ \\
$\leq 3$ & \\
Use of drugs perceive to be prophylactic treatment against malaria \\
No
\end{tabular}

Multivariate conditional logistic regression taking into account clustering effect was done

${ }^{\text {a }}$ Adjusted odds ratio (OR) on children age, mother age, mosquito net age, and using prophylactic treatment against malaria by children

cover of LLIN can mean the possession and/or the hung of LLIN in the household. The first step before the use of LLIN (sleep under an LLIN) is logically its set up. In this study, it was assumed that the move of the net every morning and every night is little compatible with its correct use. Then, the definition of SL included the hung of net and the verification of its presence in the household. In most studies, the level of bed net use was estimated according to their use the night before in order to minimize memory bias [11, 29-33] as recommended by WHO indicators for the estimation of LLIN use [34]. The last definition can lead to information bias as an LLIN may be put away. An LLIN could also be use for needs other than those it was originally intended to.

A preliminary study conducted in 2008 in the OKT area showed an adjusted OR of 0.32 (95 \% CI 0.15-0.71)
Table 4 Association between uncomplicated clinical malaria and sleeping under an LLIN adjusted on other variables, Djougou-Copargo-Ouake health district

\begin{tabular}{ll}
\hline Variables & Adjusted OR $\mathbf{9 5} \% \mathbf{~ C l})$ \\
\hline Sleeping every night under an LLIN for the 2 weeks before the survey \\
Sometimes or never & 1 \\
Every night & $0.45(0.28-0.72)^{\mathrm{a}}$ \\
& $0.84(0.64-1.10)^{\mathrm{b}}$ \\
Children age group (month) & 1 \\
$24-60$ & $0.24(0.19-0.31)$ \\
$0-23$ & \\
Mother or guardian age group (year) & $1.02(0.76-1.38)$ \\
$<25$ & 1 \\
$25-35$ & $1.41(1.06-1.87)$ \\
$\geq 35$ & 1 \\
Knowing mosquitoes transmit malaria & $0.71(0.54-0.93)$ \\
No & \\
Yes &
\end{tabular}

Multivariate conditional logistic regression taking into account clustering effect was done

a,b Adjusted OR on children's age, mother's age and knowing mosquitoes transmit malaria

a Adjusted OR for group of children whose mother have been at primary school at least

b Adjusted OR for group of children whose mother have never been at school

within only six villages and a sample size limited to 35 cases and 115 controls [12]. In the context of the Gambian National Insecticide Bed Net Programme during the second year of the intervention, a case-control study was conducted to evaluate the program's effectiveness. The adjusted OR of the association between net use and malaria morbidity was estimated at 0.41 (95 \% CI $0.18-$ 0.92), while the definition of the "use of a mosquito bed net" was not precise enough in terms of the frequency of use [9]. Another study was conducted in southern Colombia and considered a 1:3 case-control design with matching on village, age, and sex. The adjusted OR of the association between the impregnated net use the night before and malaria episodes was estimated at 0.44 (95\% CI 0.20-0.98), [11]. 


\section{Conclusion}

In the context of a mass distribution of LLIN, their use still conferred protection in up to $55 \%$ against the occurrence of clinical malaria cases in children. An LLIN stays then a relevant tool for malaria control. Social factors, the poor use and the poor condition of an LLIN can be in disfavour with its effectiveness. In areas, where LLIN coverage is assumed to be universal or targeted at highrisk populations, case-control studies should be regularly conducted to monitor the effectiveness of LLIN. The findings will help NMCP and their partners to improve the quality of malaria control taking into account the particularity of each area or region as far as possible.

\section{Authors' contributions}

GBD, CR, M-CH, FR, VC designed the study. GBD, SY, EC, AD, EEN, PMD acquired field data. GBD undertook data analysis and wrote the first draft of the paper. $A D, M-C H, F R$ and $C R$ reviewed the paper. All authors read and approved the final manuscript.

\begin{abstract}
Author details
1 UMR MIVEGEC (IRD224-CNRS5290-Universités Montpellier 1 et 2), Institut de Recherche pour le Développement (IRD), 34394 Montpellier, France. ${ }^{2}$ IRD-UMR MIVEGEC (IRD224-CNRS5290-Universités Montpellier 1 et 2), Centre de Recherche Entomologique de Cotonou (CREC), Cotonou, Benin. ${ }^{3}$ Laboratoire Evolution, Biodiversité des Arthropodes et Assainissement, Faculté des Sciences et Techniques, Université d'Abomey-Calavi, Abomey-Calavi, Bénin. ${ }^{4}$ Programme National de Lutte contre le Paludisme (PNLP), Direction Nationale de la Santé Publique, Ministère de la Santé, Cotonou, Benin. ${ }^{5}$ Laboratory of Parasitic Diseases, NIAID, NIH, 4 Center Dr, Bethesda, MD 20892-0425, USA. ${ }^{6}$ Institut Pasteur de Madagascar, Antananarivo, Madagascar. ${ }^{7}$ Institut de Recherche Biomédicale des Armées, Brétigny sur Orge, France. ${ }^{8}$ Unité de Recherche sur les Maladies Infectieuses et Tropicales Emergentes, UM 63 , CNRS 7278, IRD 198, INSERM 1095, Aix-Marseille Université, Marseille, France.
\end{abstract}

\section{Acknowledgements}

We thank populations of Djougou-Copargo-Ouake and Ouidah-KpomasseTori Bossito health districts, especially the head of family and the guardians of children for their kind support and great collaboration. Funding received from the World Bank via Booster Programme for Malaria Control in Africa and Malaria National Control Program of Benin (Reference No. 555/MS/DC/SGM/ DNPS/PNLP/PALP/DNP du 30/12/2010).

\section{Competing interests}

The authors declare that they have no competing interests.

Received: 31 July 2015 Accepted: 9 February 2016

Published online: 19 February 2016

\section{References}

1. WHO. World Malaria Report 2012. Geneva: World Health Organization; 2012. http://www.who.int/malaria/publications/world_malaria_ report_2012/en/accessed. Accessed Feb 2011.

2. Roll Back Malaria. The global strategic plan Roll Back Malaria 2005-2015. Geneva: Roll Back Malaria Partnership Secretariat; 2005. http://www. rollbackmalaria.org/multimedia/partnershippublications.html\#2013 Accessed Aug 2013

3. WHO. World Malaria Report 2011. Briefing on revised estimates of cases and deaths. Geneva: World Health Organization; 2011. http://www.who int/entity/malaria/world_malaria_report_2011/burdenestimatesbriefing2011.pdf. Accessed Feb 2011.

4. WHO. Global Malaria Programme, World Health Organization. Geneva: World Malaria Report 2014. http://www.who.int/malaria/publications/ world_malaria_report_2014/en/.
5. Lengeler C. Insecticide-treated bed nets and curtains for preventing malaria. Cochrane Database Syst Rev. 2004:2:CD000363.

6. ter Kuile FO, Terlouw DJ, Phillips-Howard PA, Hawley WA, Friedman JF, Kolczak MS, et al. Impact of permethrin-treated bed nets on malaria and all-cause morbidity in young children in an area of intense perennial malaria transmission in western Kenya: cross-sectional survey. Am J Trop Med Hyg. 2003;68(4 Suppl):100-7.

7. Ministère de la Santé. Secrétariat Général du Ministère. Direction de la Programmation et de la Prospective. Annuaire des statistiques sanitaires 2012. Cotonou: Ministère de la Santé; 2012. p. 122. http://www.beninsante.bj/documents/DPP/Annuaire_statistiques_sanitaires_2012_VF.pdf Accessed Jan 2016.

8. Valencianoa M, Kissling E, Ciancio B, Moren A. Study designs for timely estimation of influenza vaccine effectiveness using European sentinel practitioner networks. Vaccine. 2010;28:7381-8.

9. D'Alessandro U, Olaleye B, Langerock P, Bennett S, Cham K, Cham B, et al. The Gambian National Impregnated Bed Net Programme: evaluation of effectiveness by means of case-control studies. Trans R Soc Trop Med Hyg. 1997;91:638-42.

10. Masoumi AH, Motabar M, Zamani Gh ND, Vatandoost H. A case-control study of determinative factors on malaria morbidity in Minab, Jask and Roodan Counties, in Hormozgan Province, Southern Iran, 2001. Iran J Publ Health. 2003;3:14-8.

11. Alexander N, Rodríguez M, Pérez L, Caicedo JC, Cruz J, Prieto G, et al. Case-control study of mosquito nets against malaria in the Amazon region of Colombia. Am J Trop Med Hyg. 2005;73:140-8.

12. Rogier $C$, Henry M-C, Luxemburger C. Méthodes d'évaluation en phase IV de l'efficacité de la lutte anti-vectorielle : l'exemple d'une étude cas-témoins de l'impact des moustiquaires imprégnées d'insecticide à longue durée d'action après leur déploiement au Bénin. Med Trop (Mars). 2009;69:195-202.

13. Landier J, Boisier P, Fotso Piam F, Noumen-Djeunga B, Simé J, Wantong $\mathrm{FG}$, et al. Adequate wound care and use of bed nets as protective factors against Buruli Ulcer: results from a case control study in Cameroon. PLoS Negl Trop Dis. 2011;5:e1392.

14. Corbel V, N'Guessan R, Brengues C, Chandre F, Djogbenou L, Martin T, et al. Multiple insecticide resistance mechanisms in Anopheles gambiae and Culex quinquefasciatus from Benin, West Africa. Acta Trop. 2007:101:207-16.

15. Djogbénou L, Pasteur N, Bio-Bangana S, Baldet T, Irish SR, Akogbeto M, et al. Malaria vectors in the Republic of Benin: distribution of species and molecular forms of the Anopheles gambiae complex. Acta Trop. 2010;114:116-22.

16. Remoué F, Damien G, Henry M-C, Corbel V. Evaluation de l'efficacité des moustiquaires imprégnées d'insecticide à longue durée d'action et de I'utilisation communautaire des combinaisons thérapeutiques à base d'artémisinine chez les enfants de moins de cinq ans en milieu rural. Rapport final-October 2011 projet: EVA-LUT-PNLP, IRD UMR-MIVEGEC, Bénin

17. Djènontin A, Bio-Bangana S, Moiroux N, Henry MC, Bousari O, Chabi J, et al. Culicidae diversity, malaria transmission and insecticide resistance alleles in malaria vectors in Ouidah-Kpomasse-Tori district from Benin (West Africa): a pre-intervention study. Parasit Vectors. 2010;3:8.

18. Damien GB, Djènontin A, Rogier C, Corbel V, Bangana SB, Chandre F, et al. Malaria infection and disease in an area with pyrethroid-resistant vectors in southern Benin. Malar J. 2010;9:380.

19. WHO. Malaria rapid diagnostic test performance: results of WHO product testing of malaria RDTs: round 4 (ISBN 978924150472 0). Geneva: World Health Organization; 2012. p. 140p.

20. Langholz B, Goldstein L. Conditional logistic analysis for case-control studies with complex sampling. Biostatistics. 2001;2:63-84.

21. David W, Hosmer SL. Logisitic regression for matched case-control studies. Applied logistic regression. 2nd ed. Hoboken: Wiley; 2000.

22. Hawley WA, Phillips-Howard PA, ter Kuile FO, Terlouw DJ, Vulule JM, Ombok M, et al. Community-wide effects of permethrin-treated bed nets on child mortality and malaria morbidity in western Kenya. Am J Trop Med Hyg. 2003;68(4 Suppl):121-7.

23. Test procedures: for insecticide resistance monitoring in malaria vector mosquitoes. Geneva: World Health Organization; 2013. http://www.who. int/malaria/publications/atoz/9789241505154/en/. Accessed Jan 2016.

24. Baragatti M, Fournet F, Henry M-C, Assi S, Ouedraogo H, Rogier C, et al. Social and environmental malaria risk factors in urban areas of Ouagadougou, Burkina Faso. Malar J. 2009;8:13. 
25. De Silva PM, Marshall JM. Factors contributing to urban malaria transmission in sub-Saharan Africa: a systematic review. J Trop Med. 2012;2012:1-10

26. Rogier C, Henry MC, Spiegel A. Diagnostic des accès palustres en zone d'endémie: bases théoriques et implications pratiques. Med Trop (Mars). 2001;61:27-46.

27. Roucher C, Rogier C, Dieye-Ba F, Sokhna C, Tall A, Trape JF. Changing malaria epidemiology and diagnostic criteria for Plasmodium falciparum clinical malaria. PLoS One. 2012;7:e46188.

28. Corbel V, Akogbeto M, Damien GB, Djenontin A, Chandre F, Rogier C, et al. Combining vector control interventions for malaria control in pyrethroid resistance area: a cluster randomized controlled trial in Benin, West Africa. Lancet Infect Dis. 2012;12:617-26. doi:10.1016/S1473-3099(12)70081-6.

29. Mouchet J, Carnevale P, Coosemans M, Julvez J, Manguin S, RichardLenoble D, et al. Biodiversité du paludisme dans le monde. Montrouge: John Libbey Eurotest Paris; 2004. p. 351.
30. Eisele TP, Keating J, Littrell M, Larsen D, Macintyre K. Assessment of insecticide-treated bednet use among children and pregnant women across 15 countries using standardized national surveys. Am J Trop Med Hyg. 2009;80:209-14.

31. Noor AM, Kirui VC, Brooker SJ, Snow RW. The use of insecticide treated nets by age: implications for universal coverage in Africa. BMC Public Health. 2009;9:369.

32. Atieli HE, Zhou G, Afrane Y, Lee MC, Mwanzo I, Githeko AK, et al. Insecticide-treated net (ITN) ownership, usage, and malaria transmission in the highlands of western Kenya. Parasit Vectors. 2011;4:113.

33. Mohammadi M, Ansari-Moghaddam A, Raiesi A, Rakhshani F, Nikpour F, Haghdost A, et al. Baseline results of the first malaria indicator survey in Iran at household level. Malar J. 2011:10:277.

34. Anonymous. Monitoring and evaluation toolkit, HIV/AIDS, tuberculosis and malaria. WHO/World Bank/UNICEF, 2004. http://www.who.int/hiv/ pub/epidemiology/en/me_toolkit_en.pdf. Accessed Aug 2013.

\section{Submit your next manuscript to BioMed Central and we will help you at every step:}

- We accept pre-submission inquiries

- Our selector tool helps you to find the most relevant journal

- We provide round the clock customer support

- Convenient online submission

- Thorough peer review

- Inclusion in PubMed and all major indexing services

- Maximum visibility for your research

Submit your manuscript at www.biomedcentral.com/submit
() Biomed Central 